\title{
VISUALIZATION OF LINES OF RESEARCH ABOUT MENTAL HEALTH STUDIES AND DIFFERENTIAL ITEM FUNCTIONING
}

Erika Margarita Arias $\mathrm{P}^{1}{ }^{1}$., Ángela Berrío ${ }^{1}$ y Juana Gómez-Benito¹.

1. University of Barcelona

\section{Background}

Differential Item Functioning (DIF) is a technical approach to identify the presence of systematic error in the measurement of latent constructs, when we applied the same item in different populations or groups. DIF has been widely used and studied for decades in educational testing, nevertheless applications in health assessment only have receive increasing interest in the last decades.

\section{Figure 1.}

Item Characteristic Curves in an Item flagged with DIF

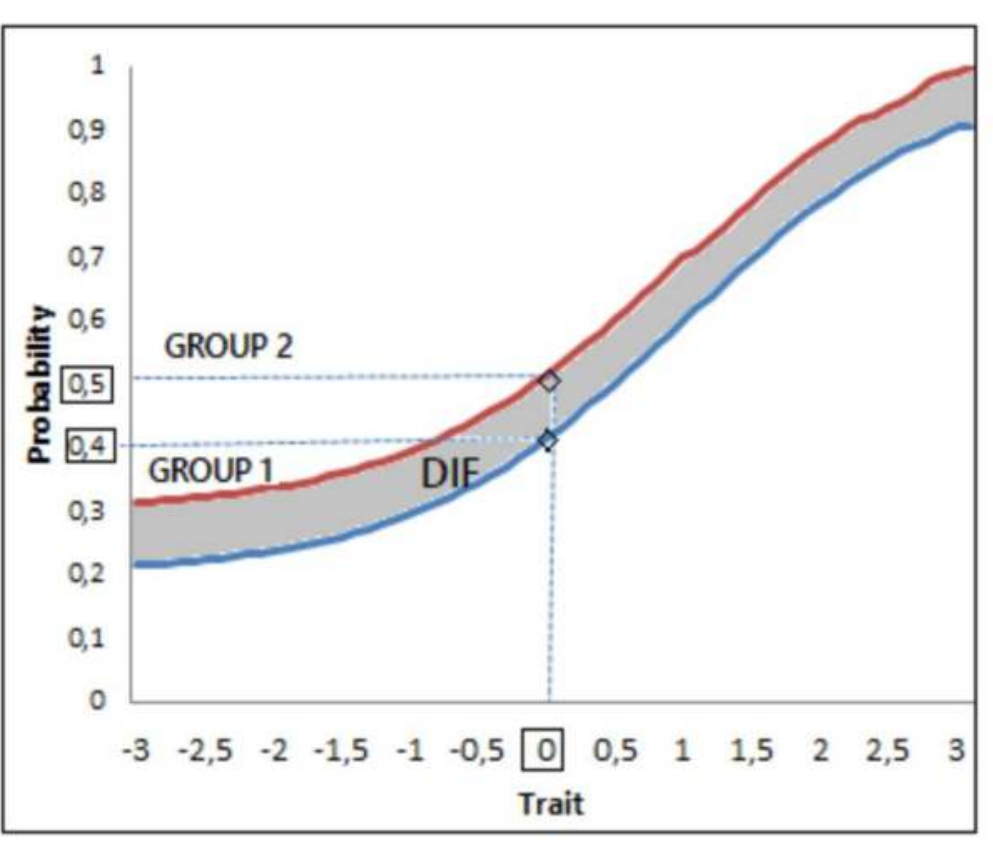

Items flagged with DIF (Figure 1) can seriously affect the validity of the tests when they are applied in different populations. In this case, the measurement is been affected by other variables, skills, or traits that are not the object of measurement, for example, gender, race, socio-economic settings, education, application place, among other contextual factors.
Method

The purpose of this paper was to analyze the scientific output about DIF in the applied area of mental health, using text mining to identify the main research perspectives in this field, by way of visualization of co-ocurrence networks of keywords extracted from scientific articles.

The search was made on the Web of Science core collection database including literature published to 2018

Following a similarity visualization methodology, distance-based maps were constructed using the VosViewer 1.6.9 software. A full counting method and a cluster resolution of 1.00 were defined as parameters for the maps.

Table 1. Application areas of item bias (DIF) studies

\begin{tabular}{lr}
\hline \multicolumn{1}{c}{ Areas of expertise } & Percentage \\
\hline Psychiatry & 18,9 \\
Health Sciences Services & 17,4 \\
Public Environmental & \\
Occupational Health & 13,9 \\
Health Policies Services & 13,3 \\
Clinical Psychology & 12,4 \\
Rehabilitation & 5,9 \\
Psychology & 5,3 \\
Gerontology & 4,7 \\
Clinical Neurology & 4,1 \\
Psychology Multidisciplinary & 4,1 \\
\hline
\end{tabular}

Results

Discussion

We analyzed 263 articles, of which 258 were in English, 4 in Spanish and 1 in Chinese. 663 keywords were obtained, of which the 120 most relevant were selected. The main application areas of studies are related with psychiatry, health services assessment, public health and clinical psychology. ( See Table 1).

Ten clusters were identified (See Figure $2)$, which are related to the application of psychometric analysis to assess the quality of tests, use of item banks (epidemiological and quality of life studies), applications of DIF analysis with different methods by a specific variable (gender, aging ), or in assessment of specific constructs (e.g cognitive impairment), cross-cultural analysis and measurement of invariance.

Figure 2. Clusters of research about DIF and mental health
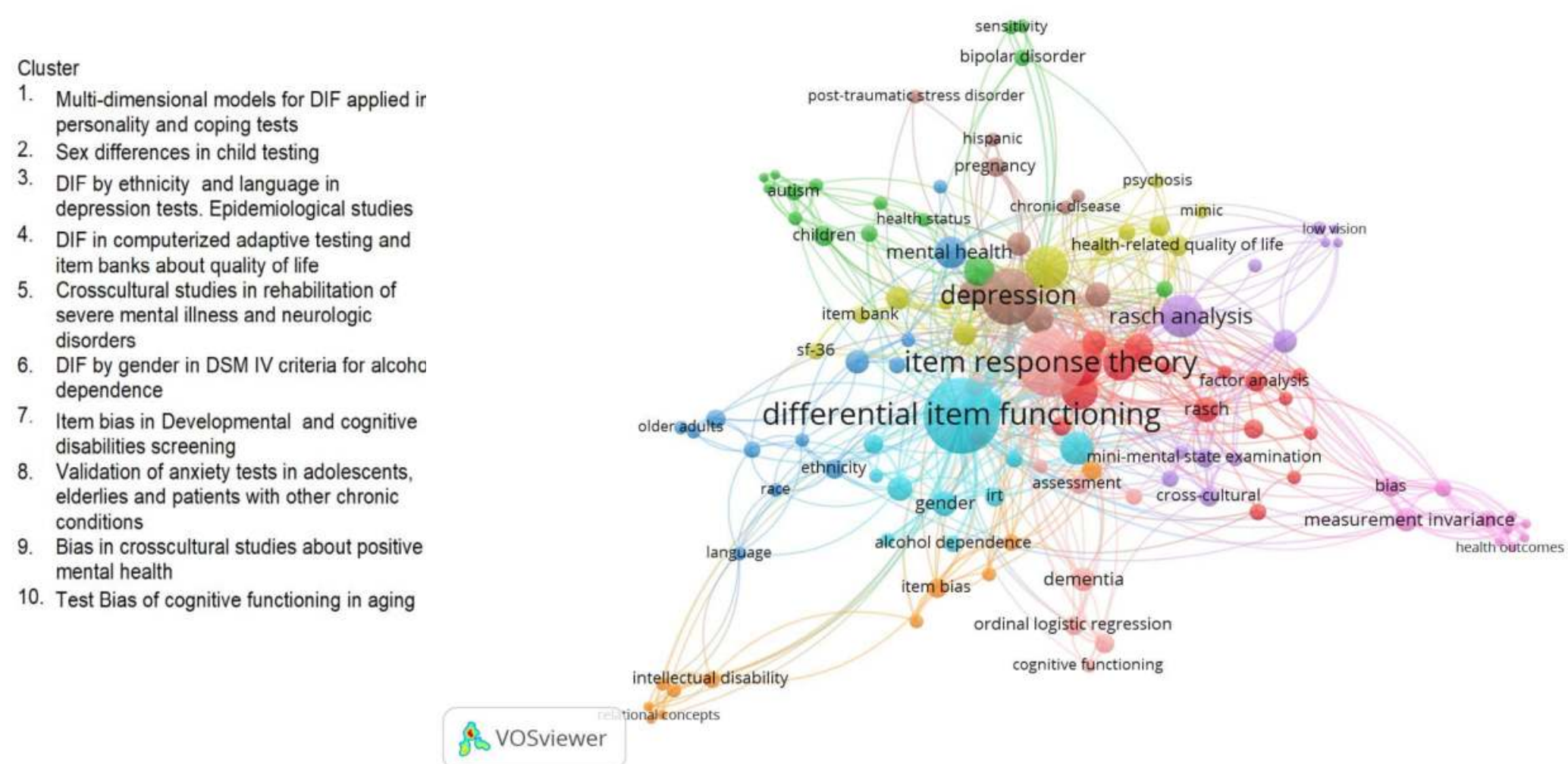

XVI European Congress of Psychology JULY 2-5 2019, MOSCOW, RUSSIA
The construction of bibliometric maps is an appropriate methodology to identify particular features about item bias research in mental health assessment. The research lines in this area were characterized by, first have a psychometric orientation; second, empirical research about specific populations by age and diagnosis, and third, use of DIF procedures in cross-cultural studies about wellness and quality of life.

This study allows us to focus on structural and contextual variables, as well as individual factors, as explanatory sources that are interacting in different areas of health assessments and hence of factors to affect the invariance of measurement. 